Federica Braga*, Elena Aloisio, Andrea Panzeri, Takahito Nakagawa and Mauro Panteghini

\title{
Analytical validation of a highly sensitive point-of-care system for cardiac troponin I determination
}

https://doi.org/10.1515/cclm-2019-0801

Received August 1, 2019; accepted September 22, 2019; previously published online October 17, 2019
Keywords: high sensitivity troponin; method evaluation; point of care testing.

\begin{abstract}
Background: Highly sensitive cardiac troponin assays (hs-cTn) are not available as point-of-care (POC) measurements. As rapid testing cannot be achieved at the expense of clinical performance, there is an urgent need to develop and rigorously validate POC hs-cTn. Konica Minolta (KM) has recently developed a surface plasmon-field enhanced fluorescence spectroscopy-based POC hs-cTn I system.
\end{abstract}

Methods: We validated the analytical characteristics of the KM POC system according to the international guidelines. Results: Limit of blank (LoB) and limit of detection (LoD) were 0.35 and $0.62 \mathrm{ng} / \mathrm{L}$, respectively, hs-cTn I concentrations corresponding to a total CV of $20 \%, 10 \%$ and $5 \%$ were $1.5,3.9$ and $11.0 \mathrm{ng} / \mathrm{L}$, respectively. Method comparison studies showed that KM calibration was successfully traced to higher-order references. Limit of quantitation (LoQ), i.e. the hs-cTn I concentration having a total error of measurement of $\leq 34 \%$, was $10.0 \mathrm{ng} / \mathrm{L}$. The upper reference limit (URL) for 600 healthy blood donors was calculated at $12.2 \mathrm{ng} / \mathrm{L}$ (90\% confidence interval [CI]: 9.2-39.2), while sex-partitioned URLs were 20.6 (males) and $10.7 \mathrm{ng} / \mathrm{L}$ (females), respectively $(\mathrm{p}<0.0001)$. KM assay measured hs-cTn I concentrations $>$ LoD in $65.7 \%$ of all reference individuals, in $76.7 \%$ of males and in $54.7 \%$ of females, respectively.

Conclusions: The KM system joins the characteristics of POC systems to the analytical performance of hs-cTn.

\footnotetext{
*Corresponding author: Federica Braga, Department of Biomedical and Clinical Sciences 'Luigi Sacco', Università di Milano, Milan, Italy; and UOC Patologia Clinica, ASST Fatebenefratelli-Sacco, Via GB Grassi 74, 20157 Milano, Italy, Phone: +390239042743, Fax: +390250319835, E-mail: federica.braga@unimi.it

Elena Aloisio, Andrea Panzeri and Mauro Panteghini: Department of Biomedical and Clinical Sciences 'Luigi Sacco', Università di Milano, Milan, Italy; and UOC Patologia Clinica, ASST Fatebenefratelli-Sacco, Milano, Italy

Takahito Nakagawa: Konica Minolta, Inc., Project Promotion Department, Product Planning Division, Precision Medicine Business Unit, Tokyo, Japan
}

\section{Introduction}

The biochemical criterion proposed to classify patients with suspected acute coronary syndrome as patients with acute myocardial infarction (AMI) is heavily predicated on an increased cardiac troponin (cTn) concentration in blood [1]. The advent of the latest generation of more sensitive cTn assays, the so-called "high-sensitivity" cTn assays (hs-cTn), has benefited the laboratory through enhanced analytical sensitivity and precision allowing to fully comply with international recommendations for the AMI definition [2]. Although cTn assays continue to improve from an analytical perspective, a performance gap still exists between central laboratory systems and point-of-care (POC) cTn testing [3]. So far, hs-cTn are not available as POC measurements and the POC cTn assays on the market lack sensitivity $[4,5]$. On the other hand, the implementation of fast track hs-cTn protocols is conditional on laboratories meeting clinically appropriate turnaround time (TAT) for reporting cTn results to clinical wards, otherwise all clinical benefits of these approaches would be lost $[6,7]$. Available guidelines recommend a TAT $\leq 60 \mathrm{~min}$ from the time of receipt of blood tubes in the laboratory to reporting [8]. To meet this requirement, laboratories have instituted dedicated pathways to quantify hs-cTn [9]. Theoretically, POC testing can be used to achieve patient care needs in situations when TAT requirements cannot be met with central laboratory systems, but the markedly lower clinical sensitivity may result in a great number of misclassified patients [10]. As rapid testing cannot be achieved at the expense of diagnostic performance, there is an urgent need to develop and rigorously validate POC hs-cTn [4].

Surface plasmon-field enhanced fluorescence spectroscopy (SPFS) is a technology with one of the highest detection sensitivities because of its low background noise [11]. Kaya et al. [12] applied this technique for a sensitive detection of prostate-specific antigen in 
serum using a plastic sensor chip. Based on their results, SPFS appeared valuable for the detection of low concentrations of biomarkers, such as cTn, in clinical samples. Recently, Konica Minolta (KM) has developed an SPFSbased POC system that provides promising perspectives to deliver hs-cTn I results of optimal quality. Here we have evaluated the conformity of the analytical characteristics of this novel POC system to current standard quality issues.

\section{Materials and methods}

\section{Equipment}

The KM SPFS POC system consists of a desktop analyzer and a plastic disposable test cartridge. The latter includes a sensor chip with immobilized capture antibody and a reagent chip, containing sample diluent, wash buffer and fluorescence-labeled detection antibody. A liquid handling system and the fluorescence detection unit are integrated into the analyzer. The assay has an analytical TAT of $\sim 15 \mathrm{~min}$. Briefly, $200 \mu \mathrm{L}$ of sample are injected into the sample well and the test cartridge is inserted into the analyzer. Here the sample is automatically diluted with sample diluent and introduced into the capture antibody-immobilized sensor chip, where cTn I in the sample is captured by immunoreaction. After a washing step, the captured cTn I is detected by the fluorescence-labeled detection antibody. Measured fluorescence is converted into marker concentration through a factory calibration curve based on four-parameter logistic regression. cTn I value is therefore adjusted with sample hematocrit (HCT) and dilution rate. In the employed prototype, HCT of each sample was obtained separately and inserted into the KM system.

The analyzer and all other materials used in the study were strictly handled according to the manufacturer's instructions. For the purpose of the study, one analyzer (prototype device v.2, serial no. 002\#13) was provided by KM to the validating site. Three lots of hs-cTn I cartridges were also provided. In all experiments, a quality control (QC) material (Liquid QC Cardiac Marker Control Complete, Cliniqa Corp.) was used daily to monitor the appropriate functionality of the analyzer and the reagents. If QC values were not within the acceptable range, the run was repeated. If QC results were consistently out of range, the study was suspended, and KM contacted to discuss the course of action.

\section{Samples}

Unless otherwise stated, measurements were carried out in singlicate. Human samples (lithium heparin whole blood [WB] or plasma, if not otherwise stated) needed for the different parts of the study were provided by the validating center. The selection of clinical samples used in the study was exclusively based on their cTn concentrations. These samples were leftovers from daily laboratory activity. When the study required measuring WB samples, the lithium heparin tube was first measured on the KM SPFS system and then centrifuged for routine activity. However, if plasma samples were required for the study, tubes were immediately centrifuged, the plasma first used for the clinical activity and leftovers measured for the study. The study was approved by the local Institutional Review Board.

\section{Study protocol}

Table 1 of the Supplementary Data that accompanies the online version of this article summarizes the items validated in the study. The methodological details are described below.

Limit of blank (LoB), limit of detection (LoD): These characteristics were assessed according to the Clinical and Laboratory Standards Institute (CLSI) EP17-A2 standard [13]. The LoB, defined as the highest measurement result that is likely to be observed for a blank sample, was estimated by 60 replicate measurements of a sample containing no cTn I in a single run and defined as $\mathrm{LoB}=$ mean $_{\text {blank }}+1.645 \mathrm{SD}_{\text {blank }}$. The Cardiac Base Diluent (Cliniqa Corp.), used as zero calibrator in the KM system, was employed for LoB estimation: this material is manufactured from healthy people's blood samples, its background level is adjusted by a heating treatment, and the lack of cTn I is verified by the manufacturer by using the Vitros ECi Troponin I ES assay (Ortho Clinical Diagnostics), with a declared LoD of $12 \mathrm{ng} / \mathrm{L}$ [14]. As the use of a contemporary assay to confirm the absence of the analyte cannot be sensitive enough, we additionally tested the material by the Architect hs-cTn I (Abbott Diagnostics) and values below the assay LoB were obtained.

The LoD, defined as the lowest amount of cTn I in a clinical sample that can reliably be detected by the assay, was obtained from 60 measurements in a single run of a pooled plasma sample with a cTn I concentration of approximately $2 \times \mathrm{LoB}$, and calculated as follows: $\mathrm{LoD}=\mathrm{LoB}+1.645 \mathrm{SD}_{\text {sample }}$. The verification of the $\mathrm{LoD}$ claim was performed according to the protocol requirements on item 7.3.1 of Ref. [13]. Ten replicates for each of two samples at the LoD claim concentration were obtained. If the observed percentage of all results that are equal to or exceed the LoB is $\geq 85 \%$, the LoD verification is deemed to be successful and the claim is taken as verified [13].

In-vitro sample stability: Five cTn I positive WB samples were analyzed immediately after collection $\left(\mathrm{T}_{0}\right)$. The samples were then divided into two aliquots. Aliquot A was kept at room temperature (RT) and analyzed after 2, 4, 6 and $8 \mathrm{~h}$. Aliquot $\mathrm{B}$ was placed at $+4^{\circ} \mathrm{C}$ and analyzed after 2, 4, 6 and $8 \mathrm{~h}$. All measurements were done in duplicate. Recovery of hs-cTn I was calculated as a percentage of the initial value obtained on fresh samples by dividing the concentrations at any storage time by the concentration at $\mathrm{T}_{0}$. The statistical analysis of trend was also done.

Linearity on dilution: The declared upper measurement limit is $50,000 \mathrm{ng} / \mathrm{L}$. Three pooled plasma were diluted with the Cardiac Base Diluent at a ratio of 1:9, 2:8, 3:7, 4:6, 5:5, 6:4, 7:3, 8:2, 9:1, 10:0 $(\times 1$ : original sample), respectively. Each point was measured in triplicate. Linearity was acceptable if there was no statistical difference between simple linear regression and 2nd order polynomial regression of all dilution points [15].

Human anti-mouse antibody (HAMA) interference: To test HAMA interference, 14 commercially available EDTA- $\mathrm{K}_{2}$ plasma samples (Promeddx), containing HAMA at high concentrations $(11.8-142.4 \mu \mathrm{g} / \mathrm{L})$ were analyzed in duplicate before and after 
treatment with a heterophilic blocking tube (HBT) (Scantibodies Laboratory, Inc.). The use of EDTA samples as an alternative to the lithium heparin samples used in this protocol was internally validated by KM (Passing-Bablok regression: EDTA $=1.01$ (95\% confidence intervals [CI]: 0.89-1.06) heparin - $1.3 \mathrm{ng} / \mathrm{L}$ [95\% CI: -2.6/0.9], $\mathrm{n}=17$ ). Treatment of samples with HBT was performed as previously described [16]. A difference between hs-cTn I values before and after HBT treatment $>25 \%$ was considered indicative of a significant HAMA interference in the evaluated assay. This criterion was chosen due to the very low cTn I concentrations in HAMA positive samples.

Assay imprecision: Six pooled plasma were prepared and stored aliquoted at $-80^{\circ} \mathrm{C}$ until use. The corresponding hs-cTn I concentrations were approximately $2 \times \mathrm{LoD}, 3 \times \mathrm{LoD}, 4 \times \mathrm{LoD}, 10 \times \mathrm{LoD}$, 99th percentile limit of the reference distribution (URL), and $\sim 200 \mathrm{ng} / \mathrm{L}$, representing a rough estimate of the value for an immediate ruling in at patient admission [17]. For each pool, two replicates per run and two runs per day were performed for 20 working days, by including two cartridge lots (10 working days per lot). Fresh frozen aliquots were thawed for each run. Total CV for each pool was calculated using the ANOVA method as described in CLSI EP5-A standard [18] and used to construct the imprecision profile. hs-cTn I concentrations corresponding to a total $\mathrm{CV}$ of $5 \%, 10 \%$ and $20 \%$ were calculated by interpolating the obtained imprecision profile.

Sample type comparison: One hundred and forty-nine fresh paired samples (WB and plasma) with detectable hs-cTn I concentrations (range: $2.0-15,316 \mathrm{ng} / \mathrm{L}$ ) were measured within $2 \mathrm{~h}$ from sample collection. The corresponding HCT was measured in paired EDTA-K samples immediately upon arrival in the laboratory on a Sysmex XN-9000 hematology system [19]. The significance of plasma vs. WB sample difference was statistically evaluated by the Wilcoxon rank sum test for paired samples.

Calibration traceability, antibody selectivity and comparison with the reference device: As recommended by the Australasian Association of Clinical Biochemists [20], the KM system calibration was traced to higher-order references. Supplementary Figure 1 describes the approach selected by KM. In particular, the Architect hs-cTn I was selected as predicated reference device and the KM calibrator was value-assigned by a manufacturer's internal procedure through a comparison experiment performed with the Architect system on cTn I positive samples. As the Architect calibrator is in turn traceable to National Institute of Standards and Technology SRM 2921 [21], the traceability to higher-order references is assured. To avoid problems in traceability implementation due to difference in antibody selectivity, the KM system uses antibodies that recognize the same epitope regions of Architect, i.e. the capture antibody immobilized on sensor chip surface is a mouse monoclonal $\left(\mathrm{IgG}_{1}\right)$, which recognizes human cTn I at 24-40 amino acids, and the detection antibody labeled with fluorescence dye is a chimeric antibody consisting of mouse derived variable region and human derived constant regions of $\operatorname{IgG}_{1}$, which recognizes human cTn I at 41-49 amino acid region.

To confirm the alignment of the KM system to the Architect assay, we compared the KM with the Architect hs-cTn I performed on an $i 2000$ platform using 139 paired samples (WB for KM and plasma for Architect) randomly selected to represent the range of cTn I values likely to be encountered in clinical practice. HCT was measured in paired EDTA- $\mathrm{K}_{3}$ samples on XN-9000 at sample arrival in laboratory and hs-cTn I on KM system immediately after. Plasma aliquots for
Architect assay were stored frozen $\left(-80^{\circ} \mathrm{C}\right)$ until measurement, done in a single run 1 month from the beginning of sample collection. The data were analyzed by Passing-Bablok regression and bias plots.

Supplementary Figure 2 summarizes the steps of different samples for different measurements carried out in sample type comparison experiment and in the comparison with the reference device.

Limit of quantitation (LoQ): To determine the LoQ, i.e. the lowest concentration of cTn I that can be quantitatively determined with stated acceptable imprecision and bias [13], the total error (TE) was assessed as bias $+1.65 \times \mathrm{CV}$ (where 1.65 is the Z-score at $95 \%$ probability), with a goal of $\leq 34 \%[8,22]$. Bias was assessed by comparison of the KM assay vs. the predicated reference device in the range of 2.0-250 ng/L and the CV derived from the imprecision profile.

Reference values: Fresh WB samples from 600 healthy blood donors (300 males and 300 females, age range: 18-70 years, sample background in Supplementary Figure 3) were analyzed. HCT was measured in paired EDTA- $\mathrm{K}_{3}$ samples on an XN-9000. The hs-cTn I URL in all individuals and in sex-partitioned groups was calculated using nonparametric determination of percentiles [23]. URL 90\% CI were calculated based on binomial probability. The presence of outliers was verified according to Reed's criterion [24]. The Mann-Whitney test was used to evaluate the between-sex differences and the age dependence of reference values was evaluated by logistic regression.

All statistical analyses were performed using MedCalc v19.0.6 software.

\section{Results}

Using the CLSI approach, the assay LoB was $0.35 \mathrm{ng} / \mathrm{L}$ and the LoD was $0.62 \mathrm{ng} / \mathrm{L}$. The 20 replicates for LoD verification gave a mean value of $0.54 \mathrm{ng} / \mathrm{L}$, with two results $<0.35 \mathrm{ng} / \mathrm{L}$. Therefore, the observed percentage of all results $\geq \mathrm{LoB}$ in the experiment was $90 \%$ and the previously determined LoD was validated.

Supplementary Figure 4 displays stability results for selected individual samples. Both at RT and $+4{ }^{\circ} \mathrm{C}$, hs-cTn I concentrations tended to significantly decrease with a mean recovery $\geq 97 \% 2 \mathrm{~h}$ and $>95 \% 4 \mathrm{~h}$ after blood collection, respectively (Figure 1). According to these data, hs-cTn I measurements by KM system should be performed within $2 \mathrm{~h}$ from sample drawing. This will assure a minimal decrease of hs-cTn I concentrations ( $\leq 3 \%)$, well inside the assay imprecision.

All three evaluated plasma pools (hs-cTn I concentrations of 240, 2251, and $6380 \mathrm{ng} / \mathrm{L}$ ) produced a perfect linear fit when their dilutions were tested for linearity (regression coefficient [R], >0.999).

Two (14.3\%) out of 14 HAMA positive samples showed a significant interference on the KM assay. However, no correlation with the HAMA concentrations in the samples was shown. The first interfered sample (HAMA concentration, $19.8 \mu \mathrm{g} / \mathrm{L}$ ) measured $5.7 \mathrm{ng} / \mathrm{L}$ 
Table 1: Summary of the assay imprecision results.

\begin{tabular}{lllllll}
\hline Sample ID & $\# \mathbf{1}$ & \#2 & \#3 & \#4 & \#5 & \#6 \\
\hline hs-cTn I concentration & 2 xLoD & $3 x$ LoD & 4xLoD & $10 x$ LoD & 99th upper reference limit & Rule in concentration \\
Mean value, ng/L & 1.13 & 1.56 & 2.28 & 5.27 & 11.8 & 193.8 \\
Within-run SD, ng/L & 0.35 & 0.17 & 0.27 & 0.28 & 0.46 & 5.28 \\
Within-run CV, \% & 30.9 & 11.0 & 11.7 & 5.3 & 3.9 & 2.7 \\
Total SD, ng/L & 0.38 & 0.27 & 0.36 & 0.38 & 0.56 & 9.01 \\
Total CV, \% & 33.5 & 17.6 & 15.7 & 7.2 & 4.8 & 4.6 \\
\hline
\end{tabular}

hs-cTn I before and $1.4 \mathrm{ng} / \mathrm{L}$ after the HBT treatment (recovery, $-72 \%$ ) and the second one (HAMA concentration, $21.9 \mu \mathrm{g} / \mathrm{L}$ ) measured $1.6 \mathrm{ng} / \mathrm{L}$ before and $0.8 \mathrm{ng} / \mathrm{L}$ after the treatment (recovery, $-50 \%$ ).

Table 1 displays the imprecision results and Figure 2 depicts the imprecision profile at cTn I low concentration range. The total $\mathrm{CV}$ at the $\mathrm{cTn}$ I concentration around URL was $<5 \%$ and cTn I concentrations corresponding to a total CV of $20 \%, 10 \%$ and $5 \%$ were $1.5,3.9$ and $11.0 \mathrm{ng} / \mathrm{L}$, respectively.

Plasma heparin gave results comparable to WB (Wilcoxon test, $p=0.289$ ) (Supplementary Figure 5) and the correlation between the two sample types was excellent (plasma $=0.96 \mathrm{WB}+0.9 \mathrm{ng} / \mathrm{L}$, coefficient of determination [R²], 0.9991).

Figure 3 shows results of the method comparison study. One sample was eliminated because it was out of the linearity limit. On the remaining 138 selected samples (hs-cTn I range: 2.0-7132 ng/L), the regression equation was as follows: $\mathrm{KM}=0.956$ Architect $-2.36 \mathrm{ng} / \mathrm{L}$; $\mathrm{R}=0.9899$. As expected, the scatter of data increased when

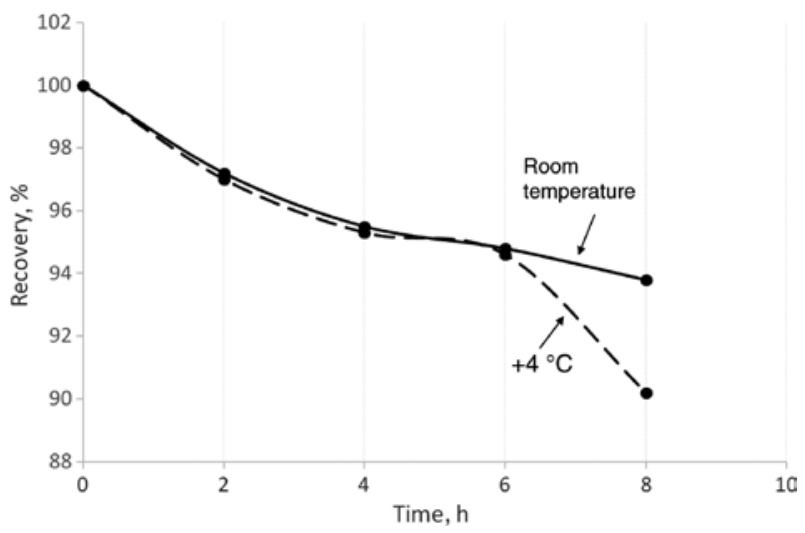

Figure 1: Summary of stability analysis of Konica Minolta POC hs-cTn I measured in whole blood.

Each point represents the mean of recovery results obtained on five samples with hs-cTn I concentrations spanning from 426 to $33,560 \mathrm{ng} / \mathrm{L}$ (see Figure 4 of the online Supplementary Data for individual sample behavior). only samples with hs-cTn I concentrations $<250 \mathrm{ng} / \mathrm{L}$ were compared (KM=0.864 Architect $-1.51 \mathrm{ng} / \mathrm{L} ; \mathrm{R}=0.9592)$. In this concentration range, $\mathrm{KM}$ results showed a mean negative bias of $-22.4 \%$ when compared with Architect, which is essentially fulfilling the bias goal (at minimum quality level) for inter-assay agreement derived from data of cTn I biological variability, i.e. $\pm 21.6 \%$ [20, 22, 25]. Despite the average negative bias, a marked positive difference was seen in two samples whose hs-cTn I concentrations (KM vs. Architect) were 9.9 vs. $3.5 \mathrm{ng} / \mathrm{L}$ and 9.3 vs. $4.9 \mathrm{ng} / \mathrm{L}$, respectively.

As reported already, the LoQ should have a maximum TE of measurement (assessed as bias $+1.65 \times \mathrm{CV}$ ) of $34 \%$. For the KM assay, this TE was obtained at hs-cTn I concentration of $10 \mathrm{ng} / \mathrm{L}$. Particularly, from imprecision evaluation, $10 \mathrm{ng} / \mathrm{L}$ showed a $\mathrm{CV}=5.2 \%$. The allowable bias at this concentration is: $34 \%=$ bias $+1.65 \times 5.2 \%$, which is bias $=34 \%-8.6 \%=( \pm) 25.4 \%$. From the regression analysis in the range of $2-250 \mathrm{ng} / \mathrm{L}(\mathrm{KM}=0.864$ Architect - $1.51 \mathrm{ng} / \mathrm{L}$ ), we can derive the Architect reference value $(11.51 / 0.864=13.3 \mathrm{ng} / \mathrm{L})$ and the corresponding $\mathrm{KM}$ system bias at $10.0 \mathrm{ng} / \mathrm{L}(-24.8 \%)$, which fulfil the bias specification reported.

Figure 4 displays the histograms of distributions of reference values. When data were verified for outliers,

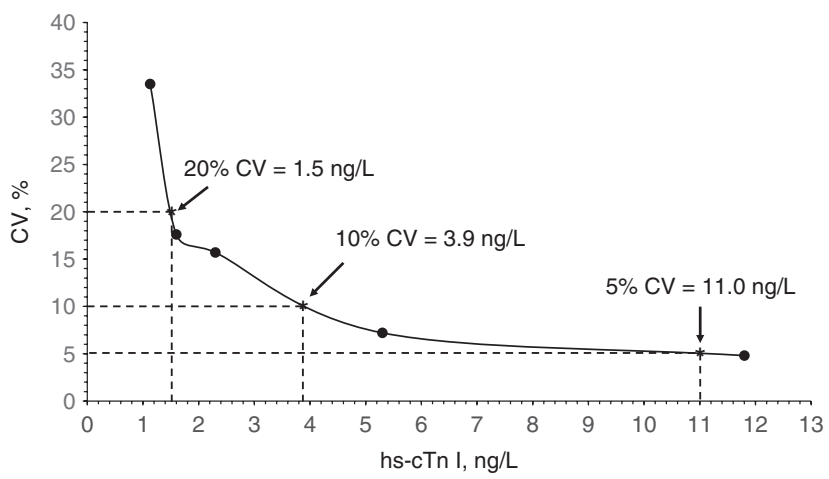

Figure 2: Imprecision profile of Konica Minolta POC hs-cTn I. Arrows show hs-cTn I concentrations with a total CV of $20 \%, 10 \%$ and $5 \%$. 
A

All data $(n=138)$
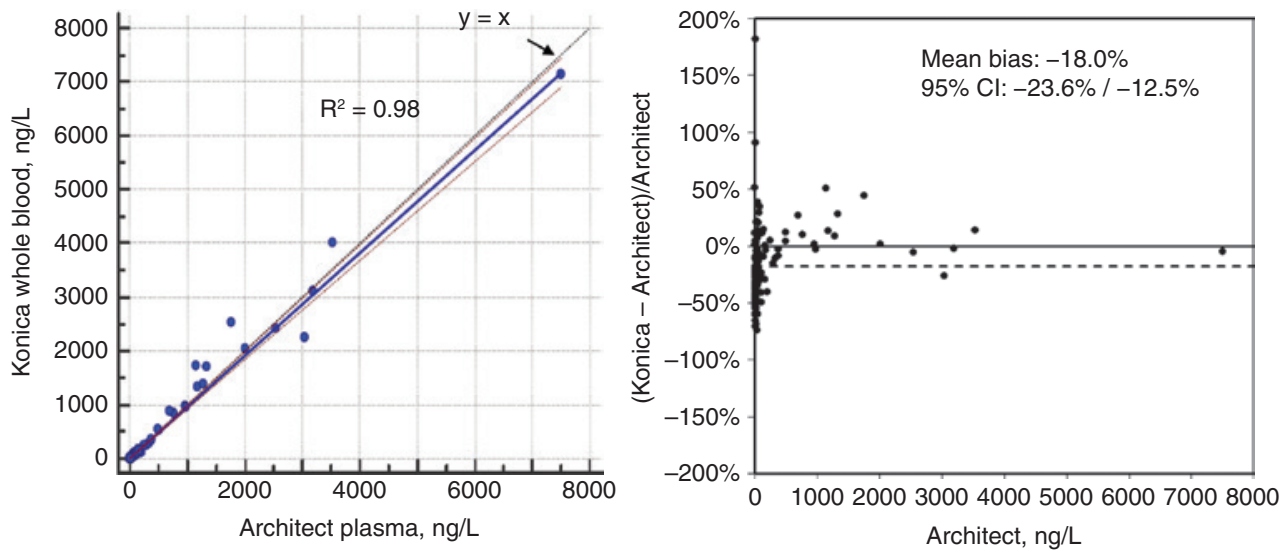

\begin{tabular}{l|ll} 
& \multicolumn{2}{|c}{$95 \% \mathrm{Cl}$} \\
\hline Slope & 0.96 & 0.92 to 0.99 \\
Intercept & $-2.4 \mathrm{ng} / \mathrm{L}$ & -3.2 to -1.8
\end{tabular}

B

hs-cTn I concentrations $<250 \mathrm{ng} / \mathrm{L}(\mathrm{n}=117)$
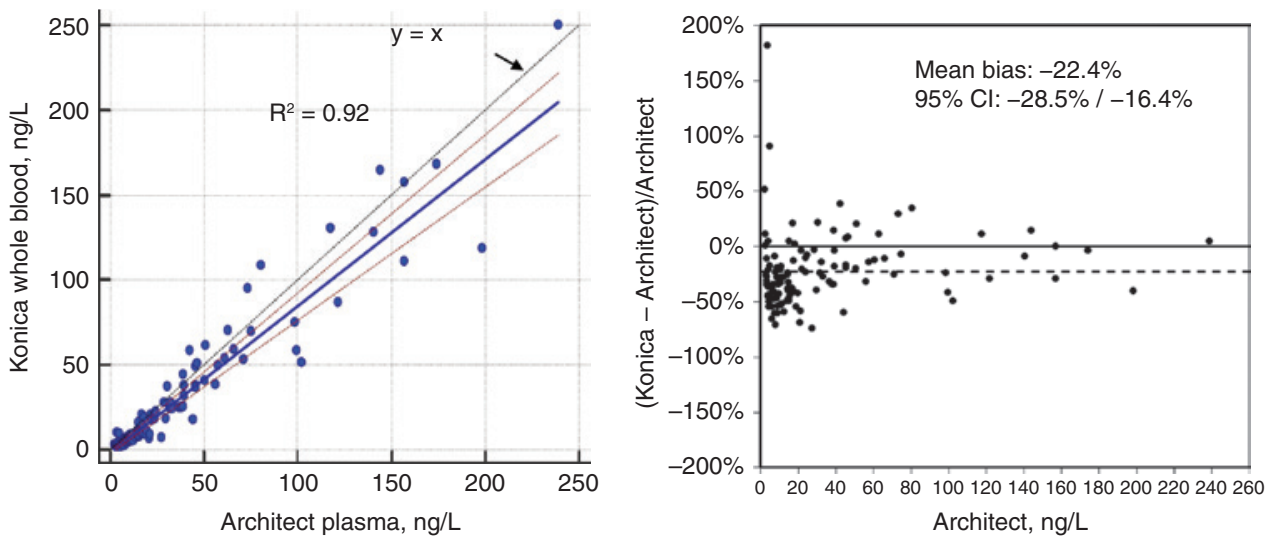

\begin{tabular}{l|ll} 
& \multicolumn{2}{|c}{$95 \% \mathrm{Cl}$} \\
\hline Slope & 0.86 & 0.79 to 0.93 \\
Intercept & $-1.5 \mathrm{ng} / \mathrm{L}$ & -2.5 to -0.5
\end{tabular}

Figure 3: Passing-Bablok regression and plots of the differences (in percentage) between Altair hs-cTn I and the designated reference device (Abbott Architect hs-cTn I).

(A) All samples $(n=138)$. (B) Samples with hs-cTn I $<250 \mathrm{ng} / \mathrm{L}(\mathrm{n}=117)$. The dashed line on the bias plots indicates the mean bias. $\mathrm{R}^{2}$, coefficient of determination; $\mathrm{Cl}$, confidence interval.

the highest data points in both sex groups $(39.2 \mathrm{ng} / \mathrm{L}$ in men and $62.8 \mathrm{ng} / \mathrm{L}$ in women) were identified as potential outliers. We reassessed hs-cTn I concentrations in these samples and obtained the same results as those initially reported, therefore excluding analytical problems. As these two particular subjects were qualified as active blood donors and no hemodynamic stress was detected by serum amino-terminal B-type natriuretic propeptide measurement (Roche Diagnostics assay), which was well below the $125 \mathrm{ng} / \mathrm{L}$ cut-off ( 24 and $20 \mathrm{ng} / \mathrm{L}$, respectively), in line with previous suggestions [23] these data points were not discarded. The URL of KM hs-cTn I for 

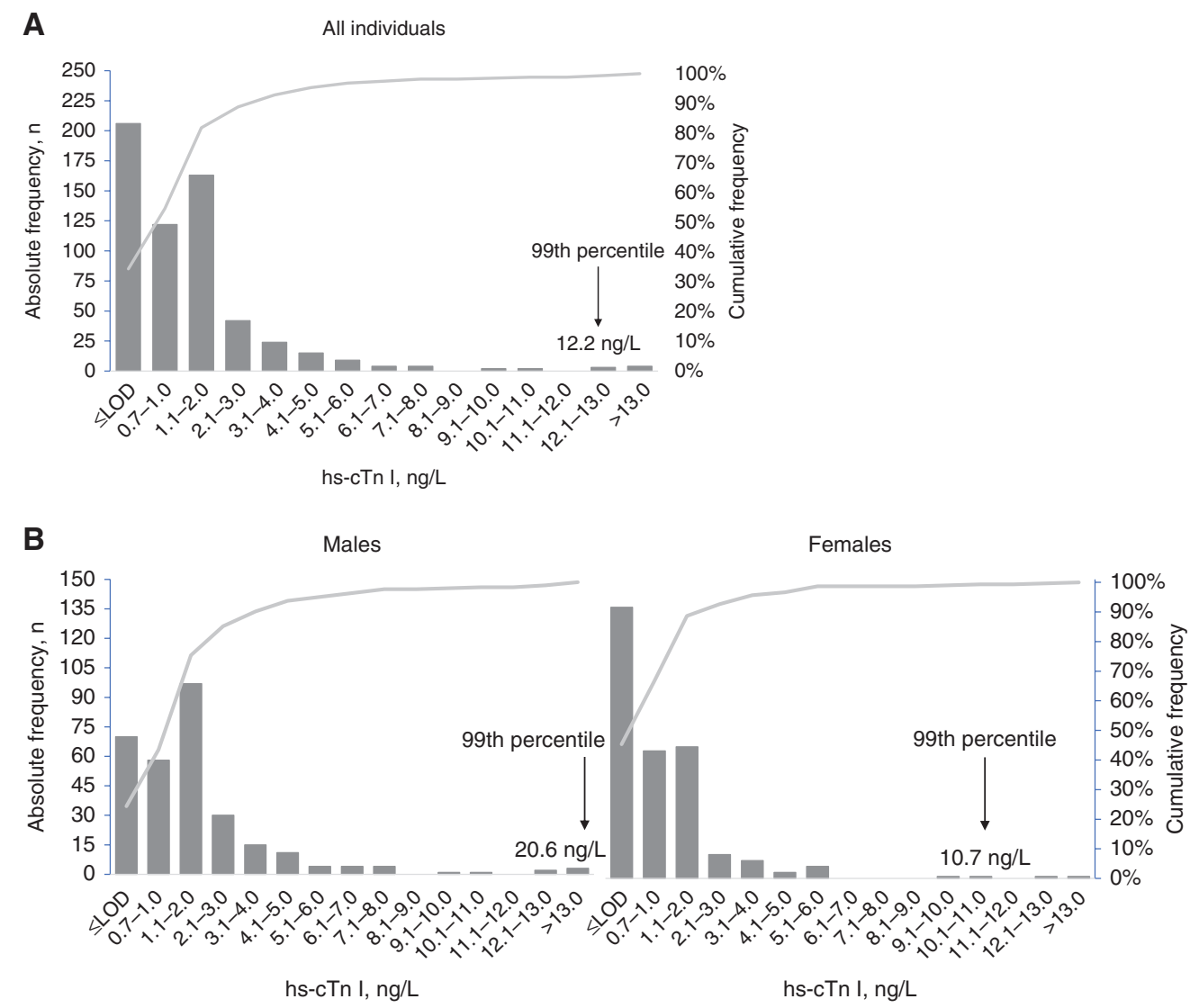

Figure 4: Histograms of distributions of reference values.

Distribution of reference values in all reference individuals (A) and in sex-partitioned groups (B).

600 healthy donors was calculated at $12.2 \mathrm{ng} / \mathrm{L}(90 \%$ CI: 9.2-39.2). However, a highly significant difference $(\mathrm{p}<0.0001)$ between values of male and female subjects was found and sex-specific URLs were derived, that are $20.6 \mathrm{ng} / \mathrm{L}$ (90\% CI: 9.2-39.2) for males and $10.7 \mathrm{ng} / \mathrm{L}$ (90\% CI: 5.5-62.8) for females. No age dependence of reference values was detected $\left(R^{2}=0.00, p=0.523\right)$. KM assay measured hs-cTn I concentrations >LoD in 394 (65.7\%) of all reference individuals, in 230 (76.7\%) of males and in 164 $(54.7 \%)$ of females, respectively.

\section{Discussion}

POC assays for cTn has been available for nearly 25 years [26]. Among the earliest commercial POC devices were qualitative lateral flow assays [27]. Later, quantitative assays were constructed using small optical readers [28]. More recently, bench-top systems have become available [7]. However, all commercially available POC cTn assays are less sensitive than central laboratory systems: this limits their use for a reliable AMI diagnosis in the emergency department. To be implemented successfully, a POC cTn assay should significantly reduce TAT without worsening analytical performance and, thus, diagnostic performance, when compared with hs-cTn performed centrally. In agreement with these premises, the new system developed by KM has been designed to meet the characteristics necessary to use it at POC without decreasing the optimal performance as obtained by the available central laboratory hs-cTn. The purpose of this study was to comprehensively validate the analytical characteristics of this system in an independent study.

Amundson and Apple [5] have summarized the desired qualities of POC cTn devices: (a) TAT $\leq 30 \mathrm{~min}$, (b) $10 \%$ CV at URL, (c) analytical specificity in the presence of other analytes potentially present in the sample, and (d) little to no sample preparation and handling to minimize the potential for errors. Devices that can achieve this level of quality represent the future of POC testing [5]. Our study results show that the KM system based on SPFS technology fulfils these requirements by joining the typical characteristics of POC systems (speed and minimal skill) to the analytical performance of hs-cTn. The LoD $<1.0 \mathrm{ng} / \mathrm{L}$ and the $\mathrm{CV}$ at URL $<5 \%$ place the system at the optimal 
level of cTn assay performance [14]. Furthermore, the optimal linearity response on dilution and the low level of HAMA interference attested appropriate measurement conditions and the validity of the immunoassay procedure [29]. Finally, its ability to quantitate cTn I above the LoD in $>50 \%$ of healthy men and women separately designs the KM system as a "high-sensitivity" assay based on the recently IFCC-AACC proposed criteria [8]. To our knowledge, only one other bench-top system, also proposed for POC use, documented a similar performance, even if much favourable data were available in the final publication when compared with a preliminary one made by the same authors [30, 31].

The US Food and Drug Administration only allows reporting of cTn assays to the LoQ [8]. However, their definition of LoQ (typically the $20 \% \mathrm{CV}$ concentration) strongly differs from the CLSI one, basically because the assay bias is ignored. In this study, we clearly showed that LoQ calculated according to CLSI criteria is much higher than the simple $20 \%$ CV (10.0 vs. $1.5 \mathrm{ng} / \mathrm{L}$ ). Ignoring bias in the LoQ estimate of hs-cTn can be very dangerous as even relatively small shifts at low marker concentrations may have a great clinical impact [32].

Since their introduction in clinical practice, cTn I assays have been plagued with a lack of standardization, which have likely contributed to widespread variability of reported cut-off concentrations [33]. Implementation of traceability concepts has been advocated as a solution [34]. Accordingly, the KM calibration was successfully traced to higher-order references and our comparison data underscored a good harmonization between the results obtained by KM system and those by the Architect assay used as predicated reference device. This is confirmed by the evidence that URLs obtained in this study for KM system closely correspond to results from independent reference studies with the Architect hs-cTn I assay [35]. Collinson et al. determined the Architect all-subject URL at $12.3 \mathrm{ng} / \mathrm{L}$, while URLs for men and women were 18.3 and $9.5 \mathrm{ng} / \mathrm{L}$, respectively [36]. Kimenai et al. found URLs of $20.0 \mathrm{ng} / \mathrm{L}$ in men and $11.0 \mathrm{ng} / \mathrm{L}$ in women, while the allsubject URL was determined to be $13.0 \mathrm{ng} / \mathrm{L}$ [37]. We want to stress that cTn elevation in acute settings should not be defined by a single value but instead by serial measurements demonstrating significant changes [38, 39]. With this regard, the high precision of the KM POC system allows for measurement of small changes in cTn I concentration over time in order to improve AMI diagnosis.

This study has some limitations. First, the study was monocentric and the generated results may not apply to other settings [40]. Second, in this study the clinical use of the KM system was not addressed: optimal analytical performance does not automatically equate to optimal clinical performance, even if it represents the mandatory precondition [38]. Clinical studies, like that recently published by Pickering et al. [41], are therefore warranted. Finally, there are potential limitations when setting one assay performance against another. In this regard, a bias of approximately 20\% between KM and Architect results may not be appropriate for permitting an integration of the two systems in clinical practice. A prospective study is required to state that the KM and Abbott assays can be used interchangeably.

Author contributions: All the authors have accepted responsibility for the entire content of this submitted manuscript and approved submission.

Research funding: Supported by an Institutional grant by Konica Minolta.

Employment or leadership: None declared.

Honorarium: None declared.

Competing interests: The funding organization(s) played no role in the study design; in the collection, analysis, and interpretation of data; in the writing of the report; or in the decision to submit the report for publication.

\section{References}

1. Thygesen K, Alpert JS, Jaffe AS, Chaitman BR, Bax JJ, Morrow DA, et al. Fourth universal definition of myocardial infarction (2018). Eur Heart J 2019;40:237-69.

2. Ferraro S, Panteghini M. Laboratory medicine as the science that underpins medicine: the "high-sensitivity" troponin paradigm. Clin Chem Lab Med 2015;53:653-64.

3. Wu AH. Recent advances in point-of-care diagnostics for cardiac markers. eJIFCC 2014;25:170-7.

4. Bruins Slot MH, van der Heijden GJ, Stelpstra SD, Hoes AW, Rutten FH. Point-of-care tests in suspected acute myocardial infarction: a systematic review. Int J Cardiol 2013;168:5355-62.

5. Amundson BE, Apple FS. Cardiac troponin assays: a review of quantitative point-of-care devices and their efficacy in the diagnosis of myocardial infarction. Clin Chem Lab Med 2015;53:665-76.

6. Ferraro S, Dolci A, Panteghini M. Fast track protocols using highly sensitive troponin assays for ruling out and ruling in non-ST elevation acute coronary syndrome. Clin Chem Lab Med 2017;55:1683-9.

7. Bingisser R, Cairns C, Christ M, Hausfater P, Lindahl B, Mair J, et al. Cardiac troponin: a critical review of the case for point-of-care testing in the ED. Am J Emerg Med 2012;30:1639-49.

8. Wu AH, Christenson RH, Greene DN, Jaffe AS, Kavsak PA, OrdonezLlanos J, et al. Clinical laboratory practice recommendations for the use of cardiac troponin in acute coronary syndrome: expert opinion from the Academy of the American Association for Clinical Chemistry and the Task Force on Clinical Applications of Cardiac Bio-Markers of the International Federation of Clinical Chemistry and Laboratory Medicine. Clin Chem 2018;64:645-55. 
9. Pasqualetti S, Birindelli S, Aloisio E, Dolci A, Panteghini M. Clinical governance remains a priority in total laboratory automation era. J Appl Lab Med 2019;4:130-4.

10. Palamalai V, Murakami MM, Apple FS. Diagnostic performance of four point of care cardiac troponin I assays to rule in and rule out acute myocardial infarction. Clin Biochem 2013;46:1631-5.

11. Arima Y, Teramura Y, Takiguchi H, Kawano K, Kotera H, Iwata $H$. Surface plasmon resonance and surface plasmon fieldenhanced fluorescence spectroscopy for sensitive detection of tumor markers. Methods Mol Biol 2009;503:3-20.

12. Kaya T, Kaneko T, Kojima S, Nakamura Y, Ide Y, Ishida K, et al. High-sensitivity immunoassay with surface plasmon fieldenhanced fluorescence spectroscopy using a plastic sensor chip: application to quantitative analysis of total prostate-specific antigen and GalNAc $\beta 1-4 \mathrm{GICNAc-linked} \mathrm{prostate-specific} \mathrm{antigen}$ for prostate cancer diagnosis. Anal Chem 2015;87:1797-803.

13. Clinical and Laboratory Standards Institute (CLSI). Evaluation of detection capability for clinical laboratory measurement procedures; approved guideline. 2nd ed. CLSI document EP17-A2, 2012.

14. Collinson PO, Saenger AK, Apple FS, on behalf of the IFCC C-CB. High sensitivity, contemporary and point-of-care cardiac troponin assays: educational aids developed by the IFCC Committee on Clinical Application of Cardiac Biomarkers. Clin Chem Lab Med 2019;57:623-32.

15. Clinical and Laboratory Standards Institute (CLSI). Evaluation of the linearity of quantitative measurement procedures: a statistical approach; approved guideline. CLSI document EP6-A, 2003.

16. Pagani F, Stefini F, Chapelle JP, Lefevre G, Graine H, Luthe H, et al. Multicenter evaluation of analytical performance of the Liaison troponin I assay. Clin Biochem 2004;37:750-7.

17. Boeddinghaus J, Nestelberger T, Badertscher P, Twerenbold R, Fitze $B$, Wussler D, et al. Predicting acute myocardial infarction with a single blood draw. Clin Chem 2019;65:437-50.

18. Clinical and Laboratory Standards Institute (CLSI). Evaluation of precision performance of quantitative measurement methods; approved guideline. 2nd ed. CLSI document EP5-A2, 2004.

19. Birindelli S, Aloisio E, Carnevale A, Brando B, Dolci A, Panteghini $M$. Evaluation of long-term imprecision of automated complete blood cell count on the Sysmex XN-9000 system. Clin Chem Lab Med 2017;55:e219-22.

20. Panteghini M. Quality requirements for troponin assays - an overview. In: Tate J, Johnson R, Jaffe A, Panteghini M, editors. Troponin Monograph 2012. Alexandria, NSW: The Australasian Association of Clinical Biochemists Inc., 2012:53-61.

21. Architect system. STAT High Sensitive Troponin-I Calibrators. REF 3P25-02, G5-6531/R01 S3P2U0. Abbott Laboratories, 2015.

22. Tate JR, Panteghini M, Koerbin G, Hickman PE, Schneider HG, Jaffe AS. Verification of the analytical characteristics of troponin assays in the laboratory - a how to guide. In: Tate J, Johnson R, Jaffe A, Panteghini M, editors. Troponin monograph 2012. Alexandria, NSW: The Australasian Association of Clinical Biochemists Inc., 2012:69-85.

23. Eggers KM, Apple FS, Lind L, Lindahl B. The applied statistical approach highly influences the 99th percentile of cardiac troponin I. Clin Biochem 2016;49:1109-2.

24. Braga F, Panteghini M. Generation of data on within-subject biological variation in laboratory medicine: An update. Crit Rev Clin Lab Sci 2016;53:313-25.

25. Wu AH, Lu AQ, Todd J, Moecks J, Wians F. Short- and long-term biological variation in cardiac troponin I measured with a high- sensitivity assay: implications for clinical practice. Clin Chem 2009;55:52-8.

26. Panteghini M. Present issues in the determination of troponins and other markers of cardiac damage. Clin Biochem 2000;33:161-6.

27. Pagani F, Serena C, Bosio C, Cuccia C, Panteghini M. Evaluation of a rapid bedside immunochromatographic assay for detection of cardiac troponin I in whole blood. Clin Chem Lab Med 2001;39:458-9.

28. Muller-Bardorff M, Rauscher T, Kampmann M, Schoolmann S, Laufenberg F, Mangold D, et al. Quantitative bedside assay for cardiac troponin $\mathrm{T}$ : a complementary method to centralized laboratory testing. Clin Chem 1999;45:1002-8.

29. Panteghini M, Gerhardt W, Apple FS, Dati F, Ravkilde J, Wu AH. Quality specifications for cardiac troponin assays. Clin Chem Lab Med 2001;39:174-8.

30. Christenson RH, Mullins K, Duh S-H. Validation of highsensitivity performance for a United States Food and Drug Administration cleared cardiac troponin I assay. Clin Biochem 2018;56:4-10.

31. Christenson RH, Duh S, Ruiz RM, Mullins K. Sex-specific 99th percentile cardiac troponin normal limits with the Medience Pathfast point-of-care cardiac biomarker analyser. Clin Chem 2017;63:S32.

32. Lyon AW, Kavsak PA, Lyon OA, Worster A, Lyon ME. Simulation models of misclassification error for single thresholds of highsensitivity cardiac troponin I due to assay bias and imprecision. Clin Chem 2017;63:585-92.

33. Panteghini M, Pagani F, Yeo KT, Apple FS, Christenson RH, Dati $F$, et al. Evaluation of imprecision for cardiac troponin assays at low-range concentrations. Clin Chem 2004;50:327-32.

34. Panteghini M. Standardization of cardiac troponin I measurements: the way forward? Clin Chem 2005;51:1594-7.

35. Kozinski M, Krintus M, Kubica J, Sypniewska G. High-sensitivity cardiac troponin assays: From improved analytical performance to enhanced risk stratification. Crit Rev Clin Lab Sci 2017;54:143-72.

36. Collinson PO, Gaze D, Goodacre S. The clinical and diagnostic performance characteristics of the high sensitivity Abbott cardiac troponin I assay. Clin Biochem 2015;48:275-81.

37. Kimenai DM, Henry RM, van der Kallen CJ, Dagnelie PC, Schram MT, Stehouwer CD, et al. Direct comparison of clinical decision limits for cardiac troponin T and I. Heart 2016;102:610-6.

38. Panteghini M. How clinical laboratories may improve their performance: the "high-sensitivity" troponin paradigm. Clin Chem 2018;64:621-3.

39. Neumann JT, Twerenbold R, Ojeda F, Sörensen NA, Chapman AR, Shah AS, et al. Application of high-sensitivity troponin in suspected myocardial infarction. N Engl J Med 2019; 380:2529-40.

40. Christenson RH, Peacock WF, Apple FS, Limkakeng AT Jr, Nowak RM, McCord J, et al. Trial design for assessing analytical and clinical performance of high-sensitivity cardiac troponin I assays in the United States: the HIGH-US study. Contemp Clin Trials Commun 2019;14:100337.

41. Pickering JW, Young JM, George PM, Watson AS, Aldous SJ, Troughton EW, et al. Validity of a novel point-of-care troponin assay for single-test rule-out of acute myocardial infarction. JAMA Cardiol 2018;3:1108-12.

Supplementary Material: The online version of this article offers supplementary material (https://doi.org/10.1515/cclm-2019-0801). 